\title{
Ethical Challenges in the Treatment of Cognitive Impairment in Aging
}

\author{
Laura B. Dunn ${ }^{1} \cdot$ Yesne Alici $^{2} \cdot$ Laura Weiss Roberts $^{1}$
}

Published online: 14 October 2015

(C) Springer International Publishing AG 2015

\begin{abstract}
The growing number of individuals with cognitive impairment, ranging from mild cognitive impairment to severe impairment experienced in Alzheimer's disease and other dementias, has become a pressing public health concern. Ethical issues related to cognitive impairment are salient in research and clinical contexts. Challenges related to the abilities of people with cognitive impairment to provide authentic informed consent or to participate in other safeguard practices meant to assure their rights and well-being are encountered in research and clinical settings. As prevention trials expand in attempts to stave off cognitive decline in individuals with specific risk biomarkers, ethical issues in research design, disclosure of findings, and other concerns at the interface of research and clinical care emerge. Additional ethical issues confront families and clinicians as patients with dementia approach the end of life. This paper reviews recent developments and empirical findings relevant to the ethical dimensions of cognitive impairment in aging, and makes specific recommendations for addressing these issues.
\end{abstract}

Keywords Aging $\cdot$ Ethics $\cdot$ Decision-making capacity Alzheimer's disease $\cdot$ Cognitive impairment $\cdot$ Informed consent $\cdot$ Surrogate consent $\cdot$ End of life

This article is part of the Topical Collection on Geropsychiatry and Cognitive Disorders of Late Life

Laura B. Dunn

laura.dunn@stanford.edu

1 Department of Psychiatry and Behavioral Sciences, Stanford University, 401 Quarry Road, Stanford, CA 94305, USA

2 Department of Psychiatry, Memorial Sloan-Kettering Cancer Center, New York, NY 10002, USA

\section{Introduction}

Absent effective treatments, it is predicted that Alzheimer's disease will affect over 120 million people worldwide by the year 2050 [1]. The numbers of older adults with mild cognitive impairment (MCI) will also expand immensely. The Centers for Disease Control recently estimated that 16 million Americans are living with cognitive impairment, broadly defined [2].

The treatment landscape for Alzheimer's disease illustrates the difficulties faced by patients, families, and providers. Alzheimer's disease has no known cure. No treatments effectively halt or slow Alzheimer's disease progression. No medications are U.S. Food and Drug Administration (FDA)-approved to treat behavioral and psychological symptoms (e.g., agitation, delusions) that commonly accompany Alzheimer's disease. End-of-life treatment dilemmas also raise difficult issues for families and providers. Fulfilling goals of care that honor the rights and foster the well-being of people with cognitive impairment, across the full spectrum of mild to severe deficits, and balancing ethical principles of autonomy, nonmaleficence, and beneficence can be extremely challenging in these clinical settings.

Improvements to this treatment landscape depend on expansion of basic, translational, and clinical research-and, ultimately, on enrolling large numbers of participants in a range of research studies. Numerous ethical issues surround this massive research endeavor-from research designs, consent issues, and disclosure of research findings to confidentiality and legal protections for research participants. Attention to these issues is crucial for research to proceed, yet relatively little work has focused on ethical dimensions of research related to cognitive impairment.

Given ethical challenges arising in both treatment and research contexts, we review recent literature pertinent to ethical implications of cognitive impairment. 


\section{Ethical Issues in Research}

Alzheimer's disease studies increasingly include, as inclusion criteria or as measures collected within protocols, an array of genetic, imaging, and other biomarkers. Programs such as the Alzheimer's Disease Neuroimaging Initiative (ADNI) are advancing the understanding of mechanisms, identifying biomarker signatures, and making preclinical diagnosis of cognitive impairment increasingly realistic [3].

As research focuses on earlier stages of disease, greater numbers of individuals with minimal or no symptoms of cognitive impairment are being enrolled in observational and clinical studies. The amyloid-binding PET ligand florbetapir was FDA-approved in 2012 for narrowly defined cases - individuals with cognitive impairment with an atypical presentation or unclear etiology. Florbetapir is not approved for preclinical testing of cognitively normal individuals. Two additional amyloid imaging agents (florbetaben and flumetamol) have since been approved for similarly narrow indications.

Human research is ethically permissible when scientifically robust and guided by the principles of respect for persons, incorporating concepts of autonomy and human dignity, of beneficence, and of justice. The ethical obligations of researchers in "returning results" to study volunteers differ from those of clinicians who return results as part of the overall therapeutic plan [4], guided by principles of beneficence and non-maleficence. Prior to florbetapir's approval, researchers did not routinely disclose amyloid results to participants, as the accuracy of this technique for detecting amyloid and predicting cognitive decline was unknown. With amyloid imaging now approved for clinical indications, researchers now confront the issue of whether and how to disclose participants' amyloid status, and clinicians must be aware of appropriate indications [5•].

Amyloid imaging detects only amyloid plaques - one of two core pathologic features of Alzheimer's disease, along with neurofibrillary tangles (undetected by amyloid-binding agents). Furthermore, amyloid plaques are found in other types of dementia (e.g., dementia with Lewy bodies) and are not uncommonly detected (up to $30 \%$ ) in cognitively normal people $[6 \cdot \bullet$. The individual risk for these people of developing $\mathrm{AD}$ is unknown $[6 \cdot \bullet]$. Participants who are amyloidpositive need to appreciate the limitations of this technique for predicting cognitive decline. No studies to date, however, have examined research participants' understanding of these predictive limitations.

Furthermore, important privacy and legal issues are raised by inclusion of $A \beta+$ individuals in research protocols that evaluate biomarkers. Patients enrolling in such trials should understand whether they will receive their amyloid test results as part of the informed consent process-i.e., whether the enrollment is "blind" or "transparent." If transparent enrollment is used, a "pre-consent" process should inform potential participants of this aspect of participation $[6 \bullet \bullet]$.
Prior to FDA approval of florbetapir, Shulman and colleagues surveyed ADNI investigators and staff regarding the return of results to participants. At that time, most ADNI investigators $(90 \%)$ did not return amyloid imaging results to participants. The majority of the survey respondents indicated, however, that FDA approval of florbetapir would influence them to return these results to participants, despite the potential for negative psychosocial effects on study volunteers and families. Further, they requested guidance regarding how to do this and emphasized the need to study the impact of returning results [7०].

Although Kim and colleagues [8•], who analyzed the ethical basis of disclosure, argue generally for transparent disclosure in the context of prevention trials, the bases for their argument deserve further discussion and empirical scrutiny. As part of the Anti-Amyloid Treatment in Asymptomatic Alzheimer's Disease (A4) Study, a secondary prevention study, Harkins et al. described the development of a process to return amyloid imaging results to cognitively normal adults within clinical trials $[6 \cdot \bullet]$. Utilizing expert input, the researchers developed consensus recommendations, including guidance for wording for brochures for participants and for the process of disclosing amyloid imaging results.

Recommendations also included having participants complete an "educational session" during the consent process regarding limitations in what is known about amyloid imaging and the potential implications of the test results. However, it was unclear whether the experts recommended excluding individuals who showed inadequate understanding (and what level of understanding should be considered adequate). Screening participants for anxiety and depression was also recommended; however, again, lack of guidance regarding the implications of a "positive" screen limits the utility of these recommendations. Given that there is no empirical evidence on which to base exclusion of these individuals from research participation, doing so could potentially bias research findings, undermining the rigor of the research itself. Further recommendations included in-person disclosure of results, telephone follow-up to evaluate the impact of disclosure, as well as "monitoring and follow-up for participants showing distress." This process of consent and disclosure is being studied within the A4 study; these results will be crucial in determining the feasibility and adequacy of such safeguards $[6 \cdot \bullet]$.

\section{Legal Protections}

Among the thorniest issues raised by advances in biomarker research are those pertaining to legal protections for individuals undergoing such testing [9••]. Arias and Karlawish 
recently detailed the problems that could face research volunteers who are tested for biomarkers that may predict subsequent risk of cognitive impairment [9••]. Amyloid imaging and other non-genetic biomarkers are not subject to restrictions on use of these data imposed by the Genetic Information Non-discrimination Act (GINA). Moreover, some aspects of the way GINA was constructed may leave patients with genetic risk for $\mathrm{AD}$, who also test positive for certain biomarkers, vulnerable to possible discrimination $[9 \cdot \bullet]$.

Gaps thus remain in legal protections for asymptomatic but biomarker-positive individuals participating in clinical trials or who may manifest early signs of cognitive impairment. As argued by these authors, policymakers should address these limitations. To our knowledge, no studies have examined the understanding, perceptions, or concerns of research participants or their close relatives (who may also be affected indirectly by such information) regarding confidentiality or legal protections related to biomarker risk information related to cognitive impairment. As a matter of justice and the positive duty to avoid foreseeable harms, such inquiry of relevant stakeholders is warranted.

\section{Surrogate Consent to Research}

Researchers who study Alzheimer's disease and other neurodegenerative disorders face the ethical issue of including patients who lack decision-making capacity in clinical studies. While surrogate consent (along with patient assent) is typically used, numerous questions remain pertaining to how surrogates approach making decisions on behalf of the cognitively impaired individual, as well as the degree of involvement that the cognitively impaired individual has, or wants to have, in the research participation decision.

In a study examining how surrogate decision-makers balance principles of substituted judgment and best interests [10•], surrogates framed their decision-making role in terms of honoring the patient's wishes and values, perceived a duty to maintain the patient's quality of life (upholding the patient's best interests), and attempted to discern the patient's current vs. premorbid preferences.

Black and colleagues interviewed both cognitively impaired individuals as well as their surrogate decision-makers in the context of research decision-making for one of six actual dementia studies [11•]. The authors found that there was frequently disagreement between the patient and their surrogate in their perspectives on how research participation decisions were made. Further studies - particularly in real-world research settings - are needed to inform best practices for ethical enrollment of these patients.

\section{Ethical Issues in Diagnosis and Treatment of Cognitive Impairment}

Three clinical stages of Alzheimer's disease progression have been described [12] in order to facilitate both research and treatment. Preclinical Alzheimer's disease refers to the presence of biomarkers that have been reliably associated with progression to Alzheimer's disease. This specific stage, however, is currently used for research and not clinical purposes, as the clinical significance of these biomarkers for a given individual cannot be determined. It is unclear, however, whether research participants, who learn they are positive for specific biomarkers, will grasp this distinction (particularly when engaged in research in a setting where they also may receive clinical care) [13•]. As alluded to above, the consent process for such trials should be optimized and studied to enhance understanding.

Given the lack of effective preventive measures for these individuals, a "diagnosis" of preclinical Alzheimer's disease is analogous to learning of one's risk for other serious diseases for which there are no treatments. For some neurodegenerative diseases (e.g., Huntington's disease), genetic testing provides deterministic, rather than probabilistic, information. In contrast, an individual who is given a preclinical Alzheimer's disease diagnosis faces uncertainty. Ethical issues in such situations therefore include tensions among ethical principles such as truth telling (honest and complete disclosure of relevant information), autonomy (the individual's right and capacity to decide what happens in their care), and non-maleficence (the professional's duty to avoid harm). As predictive abilities grow, these ethical tensions will gain increasing attention.

The term mild cognitive impairment (MCI) is used when individuals manifest mild memory impairment, have relative preservation of other cognitive domains and functional activities, and do not meet the criteria for dementia. Currently, there are no approved treatments for MCI. It remains unclear to what degree amyloid imaging will prove useful in predicting progression from MCI to Alzheimer's disease, or in monitoring response to treatments [14]. Although the implications of a diagnosis of MCI have been minimally studied, potential harms are now considered by many to be outweighed by potential benefits (e.g., planning for the future, reduced uncertainty).

Ethical issues emerge plainly with a diagnosis of Alzheimer's disease. These concern whether an individual possesses capacity for various tasks (e.g., capacity for medical decisions, financial capacity, testamentary capacity, capacity for everyday decisions), the relative risks and benefits of specific treatments, treatment discontinuation, goals of care, and issues at the end of life. 


\section{Medical Decision-Making Capacity}

Overall medical decision-making capacity is predicated on four abilities: the ability to communicate, the ability to understand, the ability to reason, and the ability to appreciate the decision at hand. Each of these abilities may be eroded progressively by conditions that cause cognitive impairment. Few studies have followed patients over time to examine medical decision-making capacity, making it difficult to predict when patients may begin to encounter difficulties. Okonkwo and colleagues addressed this gap through a longitudinal examination of medical consent capacity among patients with MCI $(n=116)$ and in healthy controls $(n=88)$ [15]. Using the Capacity to Consent to Treatment Instrument (CCTI), an instrument that uses vignettes to assess decisional capacity, the authors found declining trajectories of medical decisionmaking capacity over time on the understanding standard of capacity among patients with MCI, but not on the other standards assessed by the CCTI. As patients converted from MCI to $\mathrm{AD}$, the decline in understanding accelerated.

Based on these findings and clinical observations, there may be a critical window for attention to consent capacity. For clinicians caring for patients with MCI, and for their families, discussions of capacity-i.e., options as the patient loses capacity — could be helpful, as could educating families about specific laws (which may vary by jurisdiction) regarding who can serve as a surrogate decision-maker.

\section{Ethical Issues in Psychopharmacologic Treatment}

The primary goals of using psychopharmacological interventions (i.e., antipsychotics, antidepressants, mood stabilizers, or sedative-hypnotics) in cognitively impaired patients are to relieve suffering, enhance quality of life, minimize risks of harm to self or others, and improve the ability to communicate with family and other caregivers. The cognitively impaired are a vulnerable group from an ethical perspective, and many have overlapping sources of vulnerability-i.e., decisional compromise, chronic illness, fragile psychosocial support, and challenging economic circumstances. Moreover, the lack of adequate infrastructure and clinical services available to cognitively impaired individuals has in some cases led to overreliance on pharmacological strategies for addressing the burden of disease - rather than enhancing the quality of life and functional capacities of those affected. Therefore, strict adherence to ethical principles in prescribing for this patient population is essential.

The fundamental ethical principle at stake when treating cognitively impaired patients with a psychopharmacologic agent is "primum non nocere" (first, do no harm)-i.e., the principle of non-maleficence. Clinicians should take into consideration any potential side effects of medications such as confusion, metabolic abnormalities, cerebrovascular events, or mortality that may harm or further destabilize the patient before initiating a particular therapeutic intervention. Acting with compassion and empathy for the ill individual and balancing non-maleficence with beneficence, clinicians ideally will strive to utilize medications that will enhance the patient's welfare, while minimizing adverse effects. Because many individuals with cognitive impairment have many coexisting physical and mental conditions, moreover, it is likely that the clinician will need to carefully evaluate the potential for medication interactions or the possibility that introducing new medications may lead to a decline in other aspects of the patient's health.

Autonomy is a delicate bioethical principle in this patient population. Values, dignity, and sense of self are taken into consideration in an informed consent framework discussing the advantages, disadvantages, rationale, and alternatives of psychotropic medications with the patient or the health care proxy. The use of most psychotropic medications in patients with Alzheimer's disease, delirium, or other cognitive impairment is "off-label" and therefore a form of clinical innovation. In this context, special efforts must be undertaken to assure that the anticipated benefits outweigh the anticipated risks in making a treatment recommendation. Stated more strongly, because "off-label" uses of medications could represent a deviation from standard of care, special efforts must be undertaken to assure an optimal informed consent process. Of note, patients with mild to early-moderate-stage Alzheimer's disease, or patients with delirium, should not be presumed to lack treatment decision-making capacity. Constructive efforts to work with the cognitive strengths of the patient are imperative, such as repeated information sessions, use of visual materials, and presentation of information about proposed treatments and alternatives, including no intervention.

The use of psychopharmacological agents, in particular the use of antipsychotics, has been a topic of significant interest due to reports of increased risk of stroke and mortality with the use of these agents $[16,17]$. In 2005, the FDA issued a black box warning for atypical antipsychotics, indicating that these drugs were associated with a higher risk of mortality among elderly persons with dementia. However, these medications continue to be widely prescribed. In 2008, the warning was extended to the use of typical or first-generation antipsychotics [18].

The principles of beneficence, non-maleficence, and autonomy are in play around this topic, often competing with one another. Prescribing a medication that might help patients with symptoms that affect their quality of life and even their own or others' safety involves tensions between beneficence and nonmaleficence. Non-maleficence argues for not prescribing a medication that might harm the cognitively impaired patient, either through increased risk of side effects, or sedation, when there may be other non-pharmacologic strategies to help the 
patient. The principle of autonomy reinforces the need to obtain informed consent - in this case, from a surrogate decision-maker-when prescribing antipsychotics when the patient lacks capacity. In many circumstances, even in the context of advanced disease, patients may consent to certain aspects of their care, e.g., timing or format of medication, even if they are not fully capable of informed consent for treatment decisions. Adhering to the meaning of autonomy in such circumstances reaffirms the importance of patients consenting to what they can as an ideal of clinical care. Unfortunately, no data exist as yet regarding the extent to which families or other surrogates are involved in these day-to-day medical decisions.

In part driven by concerns regarding antipsychotic prescribing for decisionally incapable cognitively impaired patients, a recent California Superior Court ruling brought the issue of "who decides" on behalf of these patients (specifically, nursing home residents) into sharp relief [19]. This ruling held unconstitutional a 1992 law that had allowed nursing homes to make medical decisions on behalf of "mentally incompetent" residents if a team of physicians and nurses determined they were unable to do so and the resident had no one to represent them. The 2013 lawsuit, filed by an advocacy group to stop this practice, alleged that the earlier law was being used to prescribe antipsychotic drugs, place patients in restraints, as well as deny life-sustaining treatments. The implications of this recent court decision have yet to be identified. Clarifying this critical issue-who decides when the patient lacks capacity-will likely continue to be a source of ongoing ethical discussion for years to come.

\section{Ethical Issues in End-of-Life Care}

Alzheimer's disease is a long-term, progressive, and lifelimiting illness; median survival after diagnosis is 4.2 years for men and 5.7 years for women [20]. Ethical principles of autonomy and justice make it important to communicate the progressive nature of the illness to patients and families to help them prepare for end-of-life care when patients may lack decision-making capacity. Management of agitation, behavioral disturbances, delirium, pain, feeding problems, dehydration, infections, and preferred location of care should all be discussed early in the diagnosis to assist planning and decision-making. A recent study found that, among severe dementia patients who remained in the community until death, having an advance directive in the form of a living will was associated with significantly less "aggressive" care at the end of life (i.e., as evidenced in level of Medicare spending, likelihood of in-hospital death, and use of the intensive care unit), compared to similar patients without an advance directive $[21 \bullet]$.
A common ethical dilemma at the end of life is the issue of feeding. Many AD patients with moderate to severe cognitive impairment have eating difficulties. Permanent tube feeding is not recommended for persons with advanced dementia, even for those at risk for aspiration [22]. Tube feeding does not prevent aspiration, improve quality of life, or help patients gain weight [23•]; it also minimizes social contact and may increase rates of physical or chemical restraints (to prevent patients pulling out tubes).

Reducing unnecessary polypharmacy, another aspect of end-of-life care, can minimize harms by limiting side effects and drug-drug interactions, and reducing the burden associated with pill swallowing in patients with severe dementia. For example, given concerns for weight loss with cholinesterase inhibitors in dementia patients, it is difficult to argue for maintaining patients with severe dementia on those medications [24].

A comprehensive review of guidelines for palliative care in dementia patients was recently published [25••]. Further, the Nuffield Council on Bioethics published a report on ethical issues in dementia, providing insight into several of the issues discussed above [26]. Raymond and colleagues have further delineated issues involved in end-of-life care for people with dementia [27•].

Ethical dilemmas arise in caring for patients with delirium at the end of life. Delirium, often the harbinger of impending death in palliative care settings, affects up to $80 \%$ of patients with terminal illness near death [28], and causes distress in patients, families, and clinicians [29]. While diagnostic workup of delirium in the medical setting usually includes assessment of underlying causes, in the end-of-life setting, principles of non-maleficence and beneficence may conflict, arguing for an individualized approach consistent with goals of care [28]. Non-pharmacologic management strategies should be applied to patients with delirium, regardless of setting [30]. On the other hand, pharmacologic management options, including use of antipsychotics or in some cases sedating medications, are debated. Antipsychotics can help manage agitation, paranoia, and perceptual disturbances of delirium [30]. While goals of delirium management at the end of life include a patient who is calm, pain-free, and alert, delirium severity not infrequently interferes with achieving these goals.

Sedating agents may be used to minimize suffering and to provide comfort to patients. Studies have found that the compassionate and correct clinical use of sedating agents does not hasten death [31]. Although physician-assisted suicide has garnered increasing acceptance in some areas of the world (and has been legalized in several states in the USA), this practice directly contradicts the ethical and judicial code of ethics of the American Medical Association [32].

Clinicians should ask patients with life-threatening illnesses their preferences for how they would like to be treated if and when delirium occurs at the end of life. Institutional or 
hospital ethics committees, when available, provide invaluable insight into ethical challenges involved in assessment and management of delirium in the terminally ill, making case-specific assessments. Clinical indications, aligned with principles of autonomy, beneficence, non-maleficence, compassion, and, ultimately, respect for persons, are all at play in this decision-making process. A comprehensive review of the literature on ethical issues in delirium research in palliative care settings identified the key issues including involvement of palliative care patients in delirium research, capacity determination, and the mandate to respect patient autonomy and ensure maintenance of patient dignity. Designing clear, concise informed consent forms, use of concise but accurate capacity assessment instruments, and use of consent models applicable to palliative care were proposed [33*0].

Delirium in patients with underlying $\mathrm{AD}$ or other cognitive impairment represents one of the most difficult cognitive syndromes to assess and manage. Patients with cognitive impairment are at risk for developing delirium, yet the diagnosis of delirium is frequently missed in cognitively impaired individuals. While similar assessment and management principles apply to delirium patients, regardless of underlying cognitive impairment, balancing non-maleficence versus beneficence may dictate using medications that are better tolerated and at lower doses in cognitively impaired individuals.

Further research in end-of-life care of AD patients will help better address many of the ethical challenges in the care of this patient population and will bring valuable evidence to clinical care guidelines. On the other hand, as almost all patients with severe $\mathrm{AD}$ or other severe cognitive impairment lack decisionmaking capacity, a surrogate decision-maker will have to consent to clinical care or research. Although clinical decisionmaking by health care proxies is well-established, the ethical argument for proxy consenting for research participation relies on analogy and empirical data [34•].

\section{Conclusions and Recommendations}

Millions of people throughout the world live with cognitive impairment. Individuals with these conditions often experience multiple sources of vulnerability, such as chronic illness, fragile psychosocial supports, limited resources, and poor access to care. Scientific advances in understanding and prediction of cognitive impairment are already pushing ethical boundaries in both research and clinical activities. However, relatively little empirical evidence exists to help inform ethically sound research and clinical guidelines. Challenges encountered in the ecology of research and clinical settings are numerous - e.g., how to "return results" to individuals who have volunteered to participate in research posing psychological and social risks, how to ensure that individuals with cognitive impairment are allowed to participate in research that is consonant with their values, and how to assist surrogates in their role. Other issues for which data would be helpful, such as how to enhance quality of life and functional capacity and how to discuss goals of treatment and preferences regarding end-of-life care, also have been insufficiently studied.

Even in the absence of this much-needed research, however, a number of overarching recommendations can be made. In promoting autonomy and protecting those with diminished autonomy, individuals with cognitive impairment should be provided every possible opportunity to express their preferences regarding treatment, research participation, and who they would like to have make decisions on their behalf. While attention to advance directives in the clinical setting is now routine (with the goal of supporting autonomy by anticipating future incapacity), specific methods for addressing surrogate consent for research participation in the future has not taken hold in clinical settings.

Although there is strong public support for dementia research, as well as for the practice of surrogate consent for such research [35॰], there are both regulatory and practical challenges in operationalizing this support. Recommendations for moving forward with research in the face of these challenges include encouraging potential research participants to identify and assign a surrogate decision-maker. This could take the form, for example, of routinely asking patients evaluated for cognitive impairment (whether in clinical or research settings) to talk about their perspectives, hopes, and concerns regarding medical research (including whether they have participated in research before and what it was like), identify who they would want to make research decisions for them, and discuss their general values regarding research. There is also a need to support and educate surrogate decision-makers for their role in research decision-making, including acknowledging the relevance to research decisions of their knowledge of the patient's values. A greater focus on the "authenticity" of research participation decision-making by people with impaired decision-making capacity, as articulated by Kim [35•], would support individuals' abilities to make the kinds decisions of which they are likely still capable (i.e., appointing a surrogate decision-maker). Such a focus would also help alleviate the burden on surrogates of trying to discern assent from dissent — not always an easy task — and instead focus them more broadly on the patient's values and goals.

While studies to examine the ethics of biomarker disclosure in the research context are ongoing, recommendations regarding these practices can be made based on principles of autonomy, beneficence, and justice. In studies whose scientific design requires enrollment of biomarker-positive individuals, disclosure of amyloid and other biomarker status to cognitively normal adults should be done only with careful pre-consent education about the limitations regarding the predictive value of these findings. Follow-up contact should be maintained and (if needed) referrals for support for research participants who 
experience significant distress. However, exclusion of patients with depressive or anxiety symptoms from biomarker research, based on concerns about their ability to handle biomarker information, is not warranted based on present evidence, and should only be done with strong scientific justification.

Finally, institutional review boards (IRBs) should be encouraged to develop policies regarding capacity assessment and surrogate consent for research that are clear and internally consistent, that recognize the inherent difficulties in drawing bright lines between capacity and incapacity, and that assist researchers in enrolling participants using practical consent methods. In other words, the overemphasis on lengthy consent forms, which has been a recognized problem in research for years, needs to be replaced with a focus on clarity of communication and meaningful education and engagement of participants and their surrogate decision-makers. IRBs have significant leeway under the Common Rule to approve consent forms and procedures that are much less arduous and legalistic, yet most have been reluctant to experiment with or allow such procedures. The narrow focus on the consent formrather than the process and meaning of consent—remains an impediment to research conduct with cognitively impaired individuals. There is an extensive literature on the problems with typical informed consent forms for research, and there have been plenty of calls for simplification of these forms. Particularly in the context of research involving cognitively impaired individuals, IRBs should strive to make the consent process less formidable and help investigators engage participants and their surrogates in an ongoing process of research discussions. Because of the progressive nature of cognitive impairment, investigators conducting longitudinal studies will also need to revisit capacity and consent over time. However, rather than stipulating how and when this should be done, IRBs again should allow investigators to experiment with different methods for longitudinal assessment and consent. Moreover, openness on the part of IRBs to capacity- and consent-related data gathering in the context of research on cognitive impairment (rather than rigid rule imposition without an empirical basis or consensus on how investigators should go about these mandated procedures) would go a long way toward promoting investigators' interest in examining these issues. In turn, such data would help inform methods for enhancing the ethical foundation of research on cognitive impairment and dementia.

\section{Compliance with Ethical Standards}

Conflict of Interest Yesne Alici, Laura Roberts, and Laura Dunn have no disclosures relevant to this article.

Human and Animal Rights and Informed Consent This article does not contain any studies with human or animal subjects performed by any of the authors.

\section{References}

Papers of particular interest, published recently, have been highlighted as:

- Of importance

•- Of major importance

1. World Health Organization and Alzheimer's Disease International. Dementia - a public health priority. Geneva: WHO Press; 2012.

2. Centers for Disease Control and Prevention. Promoting brain health. 2011. Accessed September 1, 2015; Available from: http:// www.cdc.gov/aging/pdf/cognitive_impairment/cogImp_genAud_ final.pdf.

3. Weiner MW, Veitch DP. Introduction to special issue: Overview of Alzheimer's Disease Neuroimaging Initiative. Alzheimers Dement. 2015;11(7):730-3.

4. Roberts L, Roberts B. Psychiatric research ethics: an overview of evolving guidelines and current ethical dilemmas in the study of mental illness. Biol Psychiatry. 1999;46(8):1025-38.

5. Roberts JS, Dunn LB, Rabinovici GD. Amyloid imaging, risk disclosure and Alzheimer's disease: ethical and practical issues. Neurodegener Dis Manag. 2013;3(3):219-29. Reviews the ethical as well as practical dimensions of risk disclosure in the context of amyloid imaging trials enrolling asymptomatic individuals.

6. - Harkins $\mathrm{K}$ et al. Development of a process to disclose amyloid imaging results to cognitively normal older adult research participants. Alzheimers Res Ther. 2015;7(1):26. Describes development of a process for disclosure of amyloid imaging results to research participants; brochure template is provided.

7. Shulman MB et al. Using AD biomarker research results for clinical care: a survey of ADNI investigators. Neurology. 2013;81(13): 1114-21. Surveyed prior to the FDA approval of florbetapir, ADNI investigators provided their perspectives on disclosure of biomarker results in the context of clinical research.

8. Kim SY, Karlawish J, Berkman BE. Ethics of genetic and biomarker test disclosures in neurodegenerative disease prevention trials. Neurology. 2015;84(14):1488-94. Conceptual ethical analysis of ethics of biomarker disclosure in prevention trials.

9.• Arias JJ, Karlawish J. Confidentiality in preclinical Alzheimer disease studies: when research and medical records meet. Neurology. 2014;82(8):725-9. Highlights current legal and regulatory gaps in protections for research participants who volunteer for preclinical Alzheimer disease trials; recommends research to better identify these gaps, in order to provide guidance for policymakers and regulatory bodies.

10. Dunn LB et al. "Thinking about it for somebody else": Alzheimer's disease research and proxy decision makers' translation of ethical principles into practice. Am J Geriatr Psychiatry. 2013;21:337-45. Qualitative study examining how proxy (surrogate) decisionmakers for people with Alzheimer's disease conceive of their role with respect to making decisions on behalf of their loved one.

11. Black BS, Wechsler M, Fogarty L. Decision making for participation in dementia research. Am J Geriatr Psychiatry. 2013;21(4): 355-63. Qualitative study evaluating how research participants with dementia, and their surrogate decision-makers, perceived how decisions were made regarding research participation; found that participants with mild to moderate dementia did participate in the decision-making process for research.

12. McKhann GM et al. The diagnosis of dementia due to Alzheimer's disease: recommendations from the National Institute on AgingAlzheimer's Association workgroups on diagnostic guidelines for Alzheimer's disease. Alzheimers Dement. 2011;7(3):263-9. 
13. Lidz CW et al. Why is therapeutic misconception so prevalent? Camb Q Healthc Ethics. 2015;24(2):231-41. Proposes that therapeutic misconception (TM; i.e., inadequate appreciation by research participants of distinctions between participating in a trial and receiving typical clinical care) relates to differences in the cognitive frames of participants vs. researchers.

14. Yang L, Rieves D, Ganley C. Brain amyloid imaging-FDA approval of florbetapir F18 injection. N Engl J Med. 2012;367(10): $885-7$.

15. Okonkwo $\mathrm{OC}$ et al. Medical decision-making capacity in mild cognitive impairment: a 3-year longitudinal study. Neurology. 2008;71(19):1474-80.

16. Schneider LS, Dagerman KS, Insel P. Risk of death with atypical antipsychotic drug treatment for dementia: meta-analysis of randomized placebo-controlled trials. JAMA. 2005;294(15):1934-43.

17. Wang PS et al. Risk of death in elderly users of conventional vs. atypical antipsychotic medications. N Engl J Med. 2005;353(22): 2335-41.

18. U.S. Food and Drug Administration. Information for healthcare professionals: conventional antipsychotics. Available from: http:// www.fda.gov/Drugs/DrugSafety/PostmarketDrugSafety InformationforPatientsandProviders/ucm124830.htm. Accessed 1 September 2015.

19. Gorman A. Judge strikes California law that allowed nursing homes to make medical decisions for mentally incompetent residents. Available from: http://khn.org/news/judge-strikes-california-lawthat-allowed-nursing-homes-to-make-medical-decisions-formentally-incompetent-residents/. Accessed 1 September 2015.

20. Larson EB et al. Survival after initial diagnosis of Alzheimer disease. Ann Intern Med. 2004;140(7):501-9.

21. Nicholas LH et al. Advance directives and nursing home stays associated with less aggressive end-of-life care for patients with severe dementia. Health Aff (Millwood). 2014;33(4):667-74. Using prospectively collected data among patients with and without cognitive impairment, evaluated aggressiveness of care (as measured by total Medicare spending in 6 months preceding death, in-hospital death, and use of ICU) at the end of life. Among patients with severe dementia, advance directives were associated with less aggressive care. However, among patients with normal cognition or mild dementia, there were no differences in care between those individuals with or without advance directives.

22. Ganzini L. Artificial nutrition and hydration at the end of life: ethics and evidence. Palliat Support Care. 2006;4(2):135-43.

23. Volicer L, Simard J. Palliative care and quality of life for people with dementia: medical and psychosocial interventions. Int Psychogeriatr. 2015;27(10):1623-34. Provides recommendations for palliative care applications to dementia care. The authors underscore the need for palliative care principles to be applied to individuals with dementia, given the frequency of these patients undergoing burdensome interventions. The need to involve both patients and families in discussion about end-of-life care early in the disease trajectory is emphasized.

24. Sheffrin $\mathrm{M}$ et al. Weight loss associated with cholinesterase inhibitors in individuals with dementia in a national healthcare system. J Am Geriatr Soc. 2015;63(8):1512-8.

$25 . \bullet$ van der Steen JT et al. White paper defining optimal palliative care in older people with dementia: a Delphi study and recommendations from the European Association for Palliative Care. Palliat Med. 2014;28(3):197-209. Based on expert recommendations and iterative revision and feedback, the authors drafted and revised consensus recommendations regarding palliative care for older adults with dementia; not all recommendations achieved full consensus.

26. Nuffield Council on Bioethics. Dementia: ethical issues. 2009. Available from: http://nuffieldbioethics.org/project/dementia/. Accessed 1 September 2015.

27. Raymond $\mathrm{M}$ et al. Palliative and end of life care for people with dementia: lessons for clinical commissioners. Prim Health Care Res Dev. 2014;15(4):406-17. Literature review of peer-reviewed papers published between 2000 and 2011 focusing on palliative and end-of-life care in dementia. Identified five primary themes: caregivers' experiences, person-centered care, specific practice issues, systems factors (including ethical dilemmas), and research priorities.

28. Breitbart W, Alici Y. Agitation and delirium at the end of life: "We couldn't manage him". JAMA. 2008;300(24):2898-910. E1.

29. Breitbart W, Gibson C, Tremblay A. The delirium experience: delirium recall and delirium-related distress in hospitalized patients with cancer, their spouses/caregivers, and their nurses. Psychosomatics. 2002;43(3):183-94.

30. Breitbart W, Alici Y. Evidence-based treatment of delirium in patients with cancer. J Clin Oncol. 2012;30(11):1206-14.

31. Rietjens JA et al. Palliative sedation in a specialized unit for acute palliative care in a cancer hospital: comparing patients dying with and without palliative sedation. J Pain Symptom Manag. 2008;36(3):228-34

32. American Medical Association, Council on Ethical and Judicial Affairs. Opinion 2.211 - physician-assisted suicide. Available from: http://www.ama-assn.org/ama/pub/physician-resources/ medical-ethics/code-medical-ethics/opinion2211.page. Accessed September 7, 2015?

33.• Sweet $\mathrm{L}$ et al. Ethical challenges and solutions regarding delirium studies in palliative care. J Pain Symptom Manag. 2014;48(2):259

71. Based on a literature review as well as discussions at the Studies to Understand Delirium in Palliative Care Settings (SUNDIPS) meeting, the authors identified several key issues in research that includes palliative care patients with delirium. These issues included capacity assessment, respect for patient autonomy, and ensuring patient dignity. The authors proposed specific solutions to these issues, including a variety of modified consent models.

34. Johnson RA, Karlawish J. A review of ethical issues in dementia. Int Psychogeriatr. 2015;27(10):1635-47. Reviews ethical issues in dementia, including emerging questions related to new disease categories such as preclinical or at-risk status, and protection of research participants with cognitive impairment.

35. Kim SY. The ethics of informed consent in Alzheimer disease research. Nat Rev Neurol. 2011;7(7):410-4. Reviews empirical literature on surrogate (proxy) consent for research, and proposes that the concept of "authenticity"rather than a narrow focus on autonomy and capacityshould be incorporated into the ethical consideration of dementia research. 